\title{
Padrões de Imunofluorescência do Fator Antinuclear (FAN) em Células HEp-2 de Soros Reagentes para Anti-SSA/Ro
}

\section{Antinuclear Antibodies (ANA) Immunofluorescent Pattern's in HEp-2 Cells on Samples Positive for Anti-SSA/Ro}

\author{
Priscila Schmidt Lora $^{(1)}$, Claudia Cilene Fernandes Correia Laurino ${ }^{(2)}$, Adriana Estigarribia de Freitas ${ }^{(3)}$, \\ João Carlos T. Brenol ${ }^{(4)}$, Odirlei Montecielo ${ }^{(5)}$, Ricardo Machado Xavier ${ }^{(6)}$
}

\section{RESUMO}

Objetivo: avaliar os padrões de imunofluorescência do fator antinuclear (FAN) em soros reagentes para anticorpos anti-SSA/Ro e sua associação clínica. Método: foi realizado um estudo transversal retrospectivo, no qual foram revisadas as solicitações de anticorpos antiantígenos nucleares extraíveis (anti-ENA) encaminhadas ao SPC/HCPA no período de dois anos. Das solicitações com resultado positivo para anti-ENA identificou-se qual ou quais auto-anticorpos estavam envolvidos (anti-SSA/Ro, anti-SSB/La, anti-RNP, anti-Sm, anti-Scl-70), bem como os padrões de imunofluorescência do FAN e os quadros clínicos dos pacientes anti-SSA/Ro positivo. As técnicas usadas para detecção e identificação foram FAN por imunofluorescência indireta (IFI) em células HEp-2 e anti-ENA por hemaglutinação. Resultados: das 392 solicitações analisadas 90 eram anti-ENA positivo. Houve um predomínio do sexo feminino (94\%) (86/91) e a idade média foi de 42 anos. O anti-SSA/Ro foi o mais freqüente $(67,8 \%)(61 / 90)$, sendo que todas as amostras anti-SSA/Ro positivas eram positivas para o FAN. O padrão de imunofluorescência nuclear pontilhado fino foi o predominante $(68,9 \%)(42 / 61)$ nos pacientes com anti-SSA/Ro positivo, e o quadro clínico mais encontrado foi de lúpus eritematoso sistêmico, em 50,8\% (31/61) dos pacientes. Conclusão: o teste de FAN por IFI utilizando células HEp-2 é um bom método de triagem para detecção de auto-anticorpos anti-SSA/Ro, apresentando maior associação com o padrão nuclear pontilhado fino. Diferente do que tem sido descrito na literatura, não encontramos nenhuma amostra de pacientes com anti-SSA/Ro que tenham apresentado FAN falso-negativo na IFI. Pelo menos na nossa experiência, esses dados questionam o custo-efetividade da solicitação de rotina desse exame em pacientes FAN negativo pelo teste de IFI.

Palavras-chave: auto-anticorpo, fator antinuclear, anticorpos anti-SSA/Ro, anti-ENA.

\begin{abstract}
Objective: to evaluate the pattern at immunofluorescence of the antinuclear antibodies (ANA) detected by the indirect immunofluorescence (IIF) technique in positive samples for antiSSA/Ro autoantibody and the clinical associations. Methods: a retrospective transversal study was performed in a period of two years where the all the solicitations of testing for the presence of anti-extractable nuclear antigen (anti-ENA) antibodies delivered to the SPC/HCPA were analyzed. We selected the positive samples and identified which autoantibodies were involved (anti$S S A / R O$, anti-SSB/La, anti-RNP, anti-Sm and anti-Scl70) as well as the immunofluorescence patterns by ANA testing and the clinical associations found in the patients presenting anti-SSA/Ro positive serum. IIF was used for ANA using HEp-2 cells and hemagglutination for anti-ENA antibodies detection. Results: 90 out of the 392 solicitations analyzed were anti-ENA positive, with a predominance of women $(86 / 91-94 \%)$ and the mean age was 42 years old. The most frequent autoantibody was anti-SSA/Ro $(61 / 90-67.8 \%)$ and all samples that were anti-SSA/Ro positive were also ANA positive. Speckled nuclear immunofluorescence was the most frequent ANA pattern (42/61 - 68.9\%) among the anti-SSA/Ro positive samples and systemic lupus erythematosus was the most common clinical diagnosis (31/61-50.8\%). Conclusion: ANA testing by IIF using HEp-2 cells proved to be a good screening test for the detection of anti-SSA/Ro antibodies, that showed a strong positive association to the speckled nuclear IIF pattern. As opposed to what has been described in the literature, there was no ANA negative among the anti-SSA/Ro positive samples. At least in our experience, these data question the costeffectiveness of performing routine screening for anti-SSA/Ro antibodies in ANA negative samples by IIF testing.
\end{abstract}

Keywords: autoantibody, antibodies antinuclear, anti-SSA/Ro autoantibody, ENA.

\footnotetext{
Serviços de Reumatologia e Serviço de Patologia Clínica (SPC), Hospital de Clínicas de Porto Alegre (HCPA), Faculdade de Medicina da Universidade Federal do Rio Grande do Sul (UFRGS). Fundo de Incentivo à Pesquisa e Eventos (Fipe). Recebido em 03/10/06. Aprovado, após revisão, em 22/1 1/06.

1. Farmacêutica bioquímica, Mestranda do Programa de Pós-Graduação em Ciências Médicas, Faculdade de Medicina /UFRGS

2. Farmacêutica, Ph.D. em Ciências Aplicadas à Pediatria, Serviço de Reumatologia HCPA/UFRGS.

3. Estudante de Farmácia UFRGS

4. Professor adjunto, médico reumatologista, Ph.D. em Clínica Médica, Chefe do Serviço de Reumatologia, HCPA/UFRGS

5. Médico, Residente do Serviço de Reumatologia, HCPA/UFRGS

6. Professor adjunto, Ph.D. em Imunologia, médico reumatologista, Serviço de Reumatologia, HCPA/UFRGS.

Endereço para correspondência: Dr. Ricardo Machado Xavier, Rua Ramiro Barcelos, 2.350, sala 645, CEP 90035-003, Porto Alegre, RS, Brasil, telefone (51) 2101-8340
} e-mail: rmaxavier@hcpa.ufrgs.br. 


\section{INTRODUÇÃO}

Nas doenças reumáticas, uma das principais características é a produção de auto-anticorpos, com alta afinidade contra constituintes intracelulares e extracelulares, fazendo com que estes sejam marcadores específicos dessas doenças. No entanto, esses auto-anticorpos somente possuem significado clínico quando estão associados a outras manifestações de doença ${ }^{(1)}$.

A detecção dos auto-anticorpos tem prestado importante contribuição em diferentes aspectos: como marcadores diagnósticos, indicadores de prognóstico e na monitorização da atividade das doenças auto-imunes ${ }^{(1-3)}$.

O desenvolvimento da técnica de imunofluorescência indireta (IFI) permitiu a detecção de diversos auto-anti$\operatorname{corpos}^{(3)}$. Hoje, a pesquisa do fator antinuclear (FAN), usando como substrato células HEp-2 é a metodologia de escolha para rastreamento e identificação dos padrões de imunofluorescência à qual os diversos auto-anticorpos se associam. No entanto, o teste de FAN deve ser complementado pela pesquisa e identificação de auto-anticorpos e auto-antígenos específicos, muitos dos quais apresentam grande utilidade clínica ${ }^{(4)}$.

Apesar de FAN positivo ser um dos 11 critérios estabelecidos pelo Colégio Americano de Reumatologia para a classificação de lúpus eritematoso sistêmico (LES) $)^{(5)}$, sua presença não necessariamente indica um estado patológico. Tal situação foi demonstrada em um estudo feito em doadores de banco de sangue, em que 22,6\% (113/500) dos doadores apresentavam FAN positivo e $20,4 \%$ destes com título de $1: 80^{(6)}$.

A pesquisa de antígenos nucleares extraíveis (ENA) é usada para identificação de um grupo de auto-anticorpos específicos que inclui o anti-SSA/Ro, anti-SSB/La, antiRNP, anti-Sm, anti-Scl-70 e anti-Jo-1. Esses auto-anticorpos podem ser detectados por diversas metodologias, como contra-imunoeletroforese, immunoblot, imunodifusão, Elisa (Enzyme-Linked Immunosorbent Assay) e hemaglutinação(2). Entretanto, podemos encontrar variações nos resultados, pois essas técnicas divergem em sensibilidade e especificidade ${ }^{(7)}$.

O auto-antígeno SSA/Ro é uma ribonucleoproteína, composta de uma porção polipeptídica e outra de quatro pequenas moléculas de RNA. A junção dessas duas porções forma o complexo SSA/Ro RNAs, conhecido com hY RNAs, sendo a porção antigênica desse complexo molecular a parte polipeptídica ${ }^{(8)}$.

A maior importância clínica do anti-SSA/Ro está relacionada à sua associação com o lúpus neonatal, o bloqueio congênito cardiovascular, em crianças nascidas de mães com esse auto-anticorpo, e lúpus eritematoso cutâneo subagudo $^{(9-11)}$. Esse auto-anticorpo é um dos critérios de diagnóstico dos pacientes com síndrome de Sjögren, sendo encontrado em 50-90\% dos pacientes, geralmente também associado com outro complexo antigênico, o SSB/La. No LES é encontrado em cerca de $20-80 \%$ dos $\operatorname{casos}^{(3)}$.

Mesmo o FAN por IFI, que utiliza células HEp-2 como substrato, possuindo uma alta sensibilidade para a detecção de auto-anticorpos anti-ENA, incluindo anti-SSA/Ro, podemos encontrar pacientes com anti-SSA/Ro positivo, características de doença auto-imune e FAN negativo, conforme demonstrou Hoffman et al ${ }^{(12)}$.

Esse trabalho pretende avaliar de forma retrospectiva a correlação dos soros anti-SSA/Ro positivo com os padrões de imunofluorescência do FAN, bem como os dados clínicos desses pacientes.

\section{PACIENTES E MÉTODOS}

\section{CASUÍSTICA}

Neste trabalho foi realizado um estudo transversal retrospectivo. Foram revisadas todas as solicitações de pesquisa de anti-ENA encaminhadas ao Serviço de Patologia Clínica (SPC) do Hospital de Clínicas de Porto Alegre (HCPA), no período de janeiro de 2004 a março de 2006. Em seguida, foram selecionadas as solicitações com resultados de anti-ENA positivo, e destas se pesquisou nos prontuários qual ou quais auto-anticorpos estavam envolvidos, anti-SSA/Ro, anti-SSB/La, anti-RNP, anti-Sm e anti-Scl 70, bem como sua associação com os padrões de IFI do FAN e os dados demográficos e quadros clínicos dos pacientes anti-SSA/Ro positivo. Os diagnósticos das doenças auto-imunes foram estabelecidos conforme os critérios do Colégio Americano de Reumatologia (American College of Rheumatology)(13-17).

\section{IFI}

A IFI para células HEp-2 (Wama Diagnóstica, Brasil) foi realizada de acordo com o protocolo proposto pelo fabricante. As células foram incubadas com os soros diluídos nas seguintes titulações 1:80, 1:160, 1:320, 1:640, 1:1280 em tampão salina fosfato $\mathrm{pH}$ 7,2 (PBS) por 30 minutos em câmara úmida à temperatura ambiente. Em seguida, as lâminas foram lavadas duas vezes por 10 minutos em PBS e incubadas por 30 minutos com anticorpos secundários antiIgG humana conjugada com isotiocianato de fluoresceína 
(FITC) em câmara escura à temperatura ambiente. Após a incubação, as lâminas foram lavadas em PBS e montadas com glicerina tamponada e lamínula. A leitura foi feita em microscópio de fluorescência, modelo Olympus BX 50 sob aumento de 500 vezes $^{(3)}$.

\section{ENA POR HEMAGLUTINAÇÃO}

O teste do ENA foi realizado de acordo com o protocolo proposto pelo fabricante (Virgo/Hemagem, EUA). As hemácias foram previamente sensibilizadas com os antígenos RNP, Sm, SSA/Ro, SSB/La e Scl-70; e incubadas com soros diluídos 1:50 (50ul) em solução salina por 90 minutos à temperatura ambiente. Após a incubação, foi feita a interpretação dos resultados. Para os soros que apresentaram reação positiva nessa primeira etapa de rastreamento, foi feita a identificação dos auto-anticorpos específicos, individualmente, para os diferentes ENA.

\section{ESTATÍSTICA}

Utilizamos a estatística descritiva para apresentação dos dados demográficos e proporções de testes positivos em uma planilha Excel (Microsoft, versão 2003).

\section{RESULTADOS}

Foram analisados 392 soros de pacientes com solicitações para anti-ENA no período de janeiro de 2004 a março de 2006. Encontramos nessa amostra 90 pacientes com anti-ENA positivo e 302 negativo.

Os pacientes com resultado de anti-ENA positivo apresentaram uma faixa etária de 10 a 84 anos, com média de 42 e desvio-padrão de 18,6 anos, havendo um evidente predomínio do sexo feminino de $94 \%$ (86/90).

$\mathrm{Na}$ Tabela 1, mostramos a relação entre os testes de anti-ENA positivo e o FAN. Encontramos associação entre anti-ENA positivo e FAN positivo em 97,7\% (88/90). Todos os pacientes com anti-SSA/Ro positivo apresentaram FAN positivo. Somente dois pacientes apresentaram anti-ENA positivo e FAN negativo. O quadro clínico de um desses pacientes era paniculite, nódulos subcutâneos e alopecia (paciente anti-RNP positivo), e o do outro era LES em remissão (paciente anti-RNP e anti-Sm positivos) em tratamento com glicocorticóide.

Os padrões de imunofluorescência do FAN e as freqüências dos auto-anticorpos ENA específicos estão dispostos na Tabela 2. O auto-anticorpo anti-SSA/Ro apresentou a maior freqüência do grupo, com 67,7\% (61/90), sendo 31 soros exclusivos para anti-SSA/Ro e 30 com associação com outros auto-anticorpos. Nessas 30 associações, foram encontrados os seguintes anticorpos concomitantemente ao anticorpo anti-SSA/Ro: anti-SSB/La (26,6\%); anti-RNP (26,6\%); anti-SSB/La e anti-RNP (6,6\%); anti-RNP e antiSm $(16,6)$; anti-SSB/La e anti-Sm (10\%); anti-SSB/La, anti-RNP e anti-Sm $(6,6 \%)$; e anti-SSB/La, anti-RNP, anti$\mathrm{Sm}$ e anti-Scl-70 (6,6\%). O segundo auto-anticorpo mais freqüente foi anti-RNP, representando $48,8 \%$ da amostra, seguido por anti-SSB $/ \mathrm{La}(17,7 \%)$, anti-Sm $(16,6 \%)$ e antiScl-70 (1,1\%).

Os padrões de imunofluorescência do FAN apresentados pelas amostras anti-SSA/Ro positivo foram: nuclear pontilhado fino, nuclear pontilhado grosso, nuclear homogêneo e nuclear pontilhado pontos isolados ( $<10$ pontos). E, em oito soros anti-SSA/Ro positivo, observamos a presença de mais de um padrão, estes foram identificados como padrão misto. $\mathrm{O}$ padrão de imunofluorescência mais freqüente para anti-SSA/Ro positivo foi nuclear pontilhado fino $(42 / 61)$.

Os quadros clínicos dos pacientes anti-SSA/Ro positivo estão demonstrados na Tabela 3; LES foi o quadro clínico mais encontrado em 33 dos 61 pacientes. Somente três pacientes não apresentavam características de doença autoimune, nestes, os quadros clínicos associados eram neoplasia de mama, neoplasia de útero e quadro inflamatório.

TABELA 1

COMPARAÇÃO DOS EXAMES ANTI-ENA E FAN POR IFI EM CÉLULAS HEP-2

\begin{tabular}{l|c|c}
\hline & \multicolumn{1}{c}{$\begin{array}{c}\text { Anti-ENA + } \\
\text { Anti-SSA/Ro + }\end{array}$} & $\begin{array}{c}\text { Anti-ENA + } \\
\text { Anti-SSA/Ro - }\end{array}$ \\
\hline FAN + & 61 & 27 \\
FAN - & 0 & 2 \\
\hline
\end{tabular}

$\mathrm{FAN}=$ fator antinuclear

IFI = imunofluorescência indireta

anti-ENA = auto-anticorpos contra antígenos nucleares extraíveis

anti-SSA/Ro = auto-anticorpo

$+=$ reagente

- = não-reagente

\section{DISCUSSÃO}

Usualmente, o teste anti-ENA é solicitado para identificação de auto-anticorpos específicos, após o paciente com quadro clínico de doença auto-imune sistêmica ter apresentado um teste de FAN positivo. Muitos trabalhos têm relatado uma sensibilidade inferior a $100 \%$ das células HEp-2 na detecção de anticorpos anti-SSA/Ro ${ }^{(18-22)}$. Bossuyt et al ${ }^{(20)}$ sugere que esse fato possa acontecer por uma possível perda desse auto-antígeno durante o processo de fixação dessas células, usado na produção dos kits de FAN com células HEp-2. 
TABELA 2

Padrões de imunofluorescência do FAN em CÉlulas HEp-2 E FreQÜÊNCIAS dos AUTO-ANTICORPOS ANTi-ENA

\begin{tabular}{|c|c|c|c|c|c|}
\hline \multirow[t]{2}{*}{ Padrões de imunofluorescência do FAN } & \multicolumn{5}{|c|}{ Anti-ENA $(n=90)$} \\
\hline & $\begin{array}{c}\text { Anti-SSA/Ro } \\
(\mathrm{n}=61)^{*}\end{array}$ & $\begin{array}{l}\text { Anti-RNP } \\
(\mathrm{n}=44)^{*}\end{array}$ & $\begin{array}{c}\text { Anti-SSB/La } \\
(\mathrm{n}=16)^{*}\end{array}$ & $\begin{array}{l}\text { Anti-Sm } \\
(\mathrm{n}=15)^{*}\end{array}$ & $\begin{array}{c}\text { Anti-Scl-70 } \\
(n=1)^{*}\end{array}$ \\
\hline Nuclear pontilhado fino & 42 & 22 & 12 & 6 & 0 \\
\hline Nuclear pontilhado grosso & 6 & 6 & 1 & 4 & 0 \\
\hline Nuclear homogêneo & 4 & 3 & 2 & 0 & 0 \\
\hline Nuclear pontilhado pontos isolados ( $<10$ pontos) & 1 & 3 & 0 & 1 & 0 \\
\hline Nucleolar & 0 & 1 & 0 & 0 & 0 \\
\hline \multicolumn{6}{|l|}{ Mistos } \\
\hline Nuclear pontilhado fino e citoplasmático pontilhado & 4 & 4 & 0 & 1 & 0 \\
\hline Nuclear centromático e citoplasmático pontilhado & 2 & 2 & 0 & 1 & 0 \\
\hline Nuclear homogêneo e citoplasmático pontilhado & 1 & 1 & 1 & 1 & 1 \\
\hline Nuclear pontilhado pontos isolados (< 10 pontos) e citoplasmático pontilhado & 1 & 0 & 0 & 0 & 0 \\
\hline
\end{tabular}

FAN $=$ fator antinuclear

anti-ENA = auto-anticorpos contra antígenos nucleares extraíveis

anti-SSA/Ro = auto-anticorpo

${ }^{*}=$ os padrões de FAN estão apresentados conforme os auto-anticorpos anti-ENA. Em virtude das amostras apresentarem concomitantes auto-anticorpos, a soma dos auto-anticorpos não é o total dos pacientes anti-ENA positivos.

TABELA 3

QUADROS CLÍNICOS DOS PACIENTES ANTI-SSA/RO POSITIVOS

\begin{tabular}{|c|c|c|}
\hline Quadros clínicos & $n=61$ & Idade em anos* \\
\hline LES & 34 & $(14-84)$ \\
\hline Síndrome de Sjögren & 5 & $(26-77)$ \\
\hline LES + síndrome de Sjögren & 3 & $(48-54)$ \\
\hline Artrite reumatóide & 3 & $(53-67)$ \\
\hline Lúpus eritematoso cutâneo subagudo & 2 & $(37-50)$ \\
\hline Anemia hemolítica auto-imune + síndrome de Raynaud & 1 & 80 \\
\hline Artralgia + fibromialgia & 1 & 48 \\
\hline Artrite reumatóide juvenil & 1 & 12 \\
\hline Celulite do tronco + septicemia por Staphylococcus aureus & 1 & 52 \\
\hline Doença indiferenciada do tecido conjuntivo & 1 & 35 \\
\hline Esclerodermia linear & 1 & 10 \\
\hline Esclerose sistêmica progressiva & 1 & 47 \\
\hline Hipertireoidismo + artralgia & 1 & 57 \\
\hline Neoplasia maligna de mama & 1 & 77 \\
\hline Neoplasia maligna de útero & 1 & 34 \\
\hline Poliartralgia & 1 & 60 \\
\hline Púrpura trombocitopênica idiopática & 1 & 24 \\
\hline Síndrome antifosofolípide & 1 & 43 \\
\hline Síndrome de Sjögren + artrite reumatóide & 1 & 58 \\
\hline Total & 61 & $(10-84)$ \\
\hline
\end{tabular}

anti-SSA/Ro = auto-anticorpo

LES = lúpus eritematoso sistêmico

${ }^{*}=$ os intervalos das idades de cada patologia estão apresentados entre parênteses, em anos. 
No período de dois anos, identificamos 90 pacientes com anticorpos anti-ENA positivos testados no Serviço de Patologia Clínica do Hospital de Clínicas de Porto Alegre, maioria de mulheres (94\%), com idade média de 42 anos (desvio-padrão de 18 anos), em concordância com os achados prévios ${ }^{(23,24)}$.

Nessa amostra, o anticorpo anti-SSA/Ro foi o de maior prevalência, tendo sido identificado em 67,7\% (61/90) dos pacientes anti-ENA reagente, dados semelhantes aos relatados por Sanchez-Guerrero J et al ${ }^{(19)}$, que encontraram anti-SSA/Ro positivo em $40 \%$ de sua amostra, e por Pollock and Toh ${ }^{(21)}$, que encontraram em sua amostra $65 \%$ de anti-SSA/Ro nos anti-ENA positivos.

Observamos que, dos 61 pacientes positivos para antiSSA/Ro, 30 possuíam associação com outros auto-anticorpos. A coexistência em uma mesma doença auto-imune de diversos auto-anticorpos contra distintos constituintes de um mesmo complexo molecular já foi estudada anteriormente por $\operatorname{Tan}^{(25)}$, sugerindo que esse fato pode ser explicado pela formação de complexos supramoleculares como SSA/SSB e Sm/RNP.

Nossos resultados mostram que $97 \%$ dos pacientes anti-ENA reagentes eram também reagentes para FAN. Todos os pacientes positivos para o auto-anticorpo anti-SSA/Ro foram positivos também para o teste FAN com células HEp-2, mostrando 100\% de sensibilidade desse teste para a detecção de anti-SSA/Ro. Sendo assim, concordamos com Kavanaugh et al ${ }^{(7)}$, sugerindo que, aos pacientes com FAN negativo, não há indicação de outro teste. Contudo, encontramos dois pacientes com FAN negativo e anti-ENA positivo. Um desses pacientes apresentava anti-RNP positivo e tinha evidências de doença auto-imune, e o outro apresentava anti-RNP e anti-Sm positivos com um diagnóstico de LES em remissão com uso de glicocorticóides. Encontramos na literatura um caso em que um paciente com LES apresentava anti-RNP positivo e FAN negativo ${ }^{(12)}$.

No entanto, no passado, quando o substrato utilizado para o FAN era tecido de animal (fígado e rim de ratos), podia-se verificar FAN negativo em pacientes anti-SSA/Ro positivo devido à diminuída expressão desse antígeno quando comparado às células HEp-2(13). Porém Hoffman et $a l^{(12)}$ realizaram um estudo com 494 amostras, no qual foram utilizados dois substratos para FAN por IFI, células HEp-2 e células HEp-2 geneticamente modificadas, com superexpressão do auto-antígeno SSA/Ro $\left(H E p-2000^{\circledR}\right.$ Immunoconcepts, EUA), encontrando três pacientes com anti-SSA/Ro positivo que só apresentaram positividade para FAN por IFI quando o substrato utilizado era células HEp-2 geneticamente modificadas ${ }^{(19)}$.

Apesar de a sensibilidade do FAN para LES ser de 95-100\% $\%^{(7,25)}$, podem-se encontrar pacientes com esse diagnóstico sem um teste de FAN positivo. Ainda não se sabe se esses pacientes formariam um subgrupo de LES ou se isso seria um resultado falso-negativo de $\mathrm{FAN}^{(26)}$.

O padrão de imunofluorescência do FAN predominante dos pacientes anti-ENA positivos foi o nuclear pontilhado fino, tanto naqueles com anti-SSA/Ro positivos $(68,9 \%)$ (42/61) quanto nos outros auto-anticorpos. A correlação encontrada entre anticorpos anti-SSA/Ro e o padrão nuclear pontilhado fino já foi demonstrada por outros autores $^{(3,19,27)}$ e não deve ser usada como único parâmetro, pois esse padrão também foi o mais prevalente nas amostras sem a presença desse anticorpo, o que pode ser explicado talvez pela coexistência do anticorpo anti-SSB/La, que também costuma apresentar esse padrão de imunofluorescência.

Os quadros clínicos mais encontrados nos pacientes anti-SSA/Ro positivos foram o LES e a síndrome de Sjögren, condizendo com o que se esperava encontrar, pois essas patologias estão fortemente relacionadas com a presença desse auto-anticorpo ${ }^{(22,27,28)}$

É importante ressaltar que em nosso trabalho foi utilizada hemaglutinação para a detecção de anticorpos anti-ENA, podendo assim diferir na sensibilidade e especificidade de outros métodos (Elisa, contra-imunoeletroforese, imunodifusão etc.), como já foi relatado por inúmeros autores ${ }^{26,29}$ ${ }^{33)}$. Novas pesquisas sobre essas diferentes metodologias que não só comparem técnicas de detecção dos auto-anticorpos, mas que também analisem os pacientes quanto aos seus dados clínicos e acompanhem o curso das doenças nos pacientes, são necessárias para uma melhor utilização das tecnologias disponíveis hoje $\mathrm{e}^{(34)}$.

O teste de FAN em células HEp-2 por IFI mostrou ser um bom método de rastreamento para a detecção de autoanticorpos anti-SSA/Ro, apresentando maior associação com o padrão nuclear pontilhado fino e com os quadros clínicos de LES e síndrome de Sjögren.

\section{AGRADECIMENTOS}

Agradecemos aos funcionários do Serviço de Reumatologia e do Serviço de Patologia Clínica do Hospital de Clínicas de Porto Alegre, especialmente às bioquímicas Marta Bergman Senger, Maria Clara Medina Correa e Dra. Carolina Sichinger Moura de Souza.

Declaramos a inexistência de conflitos de interesse. 


\section{REFERÊNCIAS}

1. Tan EM: Autoantibodies in Phatology and Cell Biology. Cell 67: 841-2, 1991.

2. Phan TG, Wong RC, Adelstein S: Autoantibodies to extractable nuclear antigens: making detection and interpretation more meaningful. Clin Diagn Lab Immunol 9: 1-7, 2002.

3. von Muhlen CA, Tan EM: Autoantibodies in the diagnosis of systemic rheumatic diseases. Semin Arthritis Rheum 24: 32358,1995

4. Kelley WN: Textbook of rheumatology. 5. ed. Philadelphia: Editora WB Saunders, 1997.

5. Hochberg MC: Updating the American College of Rheumatology revised criteria for the classification of systemic lupus erythematosus. Arthritis Rheum 40: 1725, 1997.

6. Fernandez SA, Lobo AZ, Oliveira ZN, Fukumori LM, AM Pr, Rivitti EA: Prevalence of antinuclear autoantibodies in the serum of normal blood dornors. Rev Hosp Clin Fac Med São Paulo 58: 315-9, 2003.

7. Kavanaugh A, Tomar R, Reveille J, Solomon DH, Homburger HA: Guidelines for clinical use of the antinuclear antibody test and tests for specific autoantibodies to nuclear antigens. American College of Pathologists. Arch Pathol Lab Med 124: 71-81, 2000

8. Macrae IJ, Doudna JA: Ro's role in RNA reconnaissance. Cell 20: 495-6, 2005

9. Chen X, Wolin SL: The Ro $60 \mathrm{kDa}$ autoantigen: insights into cellular function and role in autoimmunity. J Mol Med 82: 2329, 2004.

10. Jaeggi ET, Fouron JC, Silverman ED, Ryan G, Smallhorn J, Hornberger LK: Transplacental fetal treatment improves the outcome of prenatally diagnosed complete atrioventricular block without structural heart disease. Circulation 110: 1542-8, 2004

11. Srivastava M, Rencic A, Diglio G, et al: Drug-induced, Ro/SSApositive cutaneous lupus erythematosus. Arch Dermatol 139: 45-9, 2003.

12. Hoffman IEA, Peene I, Veys EM, De Keyser F: Detection of specific antinuclear reactivities in patients with negative antinuclear antibody immunofluorescence screening tests. Clinical Chemistry 48: 2171-6, 2002.

13. Arnett FC, Edworthy SM, Bloch DA, et al: The American Rheumatism Association 1987 revised criteria for the classification of rheumatoid arthritis. Arthritis Rheum 31: 315-24,1988.

14. Bohan A, Peter JB: Polymyositis and dermatomyositis (first of two parts). N Engl J Med 292: 344-7, 1975.

15. Bohan A, Peter JB: Polymyositis and dermatomyositis (second of two parts). N Engl J Med 292: 403-7, 1975.

16. Fox RI, Robinson CA, Curd JG, Kozin F, Howell FV: Sjogren's syndrome. Proposed criteria for classification. Arthritis Rheum 929: 577-85, 1986.

17. Masi AT, Rodnan GP, Medsger TA Jr, et al: Preliminary criteria forthe classification of systemic sclerosis (scleroderma). Arthritis Rheum 23: 581-90, 1980.

18. Kozin F, Fowler M, Koethe SM: A comparison of the sensitivities and specificities of different substrates for the fluorescent antinuclear antibody test. Am J Clin Pathol 74: 785-90, 1980.
19. Sanchez-Guerrero J, Lew RA, Fossel AH, Schur PH: Utility of anti-Sm, anti-RNP, anti-Ro/SS-A, and anti-La/SS-B (extractable nuclear antigens) detected by enzyme-linked immunosorbent assay for the diagnosis of systemic lupus erythematosus. Arthritis Rheum 39: 1055-61, 1996.

20. Bossuyt X, Frans J, Hendrickx A, Godefridis G, Westhovens R, Marien G: Detection of anti-SSA antibodies by indirect immunofluorescence. Clin Chem 50: 2361-9, 2004.

21. Pollock $\mathrm{W}$, Toh $\mathrm{BH}$ : Routine immunofluorescence detection of Ro/SS-A autoantibody using HEp-2 cells transfected with human 60 kDa Ro/SS-A. J Clin Pathol 52: 684-7, 1999.

22. Peene I, Van Ael W, Vandenbossche M, Vervaet T, Veys E, De Keyser F: Sensitivity of the HEp-2000 substrate for the detection of anti-SSA/Ro60 antibodies.Clin Rheumatol 19: 291-5, 2000.

23. Kang I, Siperstein R, Quan T, Breitenstein ML: Utility of age, gender, ANA titer and pattern as predictors of anti-ENA and -dsDNA antibodies.Clin Rheumatol 23: 509-15, 2004.

24. Craig WY, Ledue TB, Johnson AM, Ritchie RF: The distribution of antinuclear antibody titers in "normal" children and adults. J Rheumatol 26: 914-9, 1999.

25. Tan EM: Autoantibodies and Autoimmunity: A Three-Decade Prespecive. An NY Acad Sci 815: 1-14, 1997.

26. Egner W: The use of laboratory tests in the diagnosis of SLE. J Clin Pathol 53: 424-32, 2000.

27. Peene I, Meheus L, Veys EM, De Keyser F: Detection and identification of antinuclear antibodies (ANA) in a large and consecutive cohort of serum samples referred for ANA testing. Ann Rheum Dis 60: 1131-6, 2001.

28. Peene I, Meheus L, De Keyser S, Humbel R, Veys EM, De Keyser F: Anti-Ro52 reactivity is an independent and additional serum marker in connective tissue disease. Ann Rheum Dis 61: 929-33, 2002.

29. James K, Carpenter AB, Cook L, Marchand R, Nakamura RM: Development of the antinuclear and anticytoplasmic antibody consensus panel by the Association of Medical Laboratory Immunologists. Clin Diagn Lab Immunol 7: 436-43, 2000.

30. Lock RJ, Unsworth DJ: Antibodies to extractable nuclear antigens. Has technological drift affected clinical interpretation? J Clin Pathol 54: 187-90, 2001.

31. Llorente MJ, Jimenez J, Gonzalez C, et al: Biochemistry Commission for Immunological Diseases of the Spanish Society of Clinical Chemistry. Effectiveness of different methods for anti-Sm antibody identification. A multicentre study. Clin Chem Lab Med 43: 748-52, 2005.

32. Orton SM, Peace-Brewer A, Schmitz JL, Freeman K, Miller WC, Folds JD: Practical evaluation of methods for detection and specificity of autoantibodies to extractable nuclear antigens. Clin Diagn Lab Immunol 11: 297-301, 2004.

33. Welin Henriksson E, Hansson H, Karlsson-Parra A, Pettersson I: Autoantibody profiles in canine ANA-positive sera investigated by immunoblot and Elisa. Vet Immunol Immunopathol 61: 157-70, 1998.

34. Damoiseaux JG, Tervaert JW: From ANA to ENA: how to proceed? Autoimmun Rev 5: 10-7, 2006. 